Short communication

\title{
CLINICAL AND HISTOPATHOLOGICAL STUDY ON REPRODUCTIVE LESIONS CAUSED BY PASTEURELLA MULTOCIDA TYPE B2 IMMUNOGENS IN BUFFALO HEIFERS
}

\author{
H. H. IBRAHIM ${ }^{1,2}$, F. F. A. JESSE ${ }^{1,3}$, Y. ABBA ${ }^{4}$, E. L. T. CHUNG ${ }^{1}$, A. D. MARZA ${ }^{1,5}$,

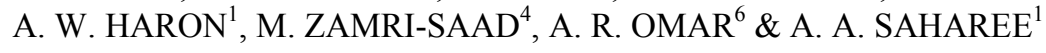 \\ ${ }^{1}$ Department of Veterinary Clinical Studies; ${ }^{3}$ Research Centre for Ruminant \\ Diseases; ${ }^{4}$ Department of Veterinary Pathology and Microbiology; Faculty of Ve- \\ terinary Medicine, Universiti Putra Malaysia, Serdang, Malaysia; ${ }^{2}$ Babil Technical \\ Institute, $\mathrm{Al}$ Furat $\mathrm{Al}$-Awsat Technical University, Iraq; ${ }^{5}$ Department of Veterinary \\ Internal Medicine, College of Veterinary Medicine, Al-Qasim Green University, \\ Iraq, ${ }^{6}$ Institute of Bioscience, Universiti Putra Malaysia, Serdang, Malaysia
}

\section{Summary}

Ibrahim, H. H., F. F. A. Jesse, Y. Abba, E. L. T. Chung, A. D. Marza, A. W. Haron, M. Zamri-Saad, A. R. Omar \& A. A. Saharee, 2018. Clinical and histopathological study on reproductive lesions caused by Pasteurella multocida type B2 immunogens in buffalo heifers. Bulg. J. Vet. Med., 21, No 2, 232-241.

\begin{abstract}
Haemorrhagic septicaemia (HS) is a globally renowned disease that affects cattle and buffaloes. Its effects on the reproductive system have not been previously studied. The present study evaluated the pathological responses in pre-pubertal female buffaloes infected with immunogens; lipopolysaccharide (LPS) and outer membrane protein (OMP) derived from P. multocida type B:2. Fifteen healthy pre-pubertal female buffaloes were divided into 5 equal groups. Buffaloes in groups 1 and 2 were intravenously and orally inoculated with $P$. multocida LPS, while those in groups 3 and 4 were subcutaneously and orally inoculated with P. multocida OMP. Buffaloes in group 5 were inoculated with sterile phosphate buffered saline (negative control). During the post infection period, all buffaloes were examined for clinical signs throughout 21 days and surviving animals were euthanised for postmortem evaluation. Histopathological evaluation of buffaloes inoculated intravenously with LPS showed a significantly higher occurrence $(\mathrm{P}<0.05)$ of necrosis and degeneration in the uterine horn and supramammary glands . Following inoculation with OMP, haemorrhage and congestion was significantly higher $(\mathrm{P}<0.05)$ in the ovaries, cervix, vagina, mammary and supra-mammary glands of buffaloes inoculated subcutaneously, while necrosis and degeneration and inflammatory cell infiltration in different organs was also significantly increased in the same group. The findings showed that both $P$. multocida bacterial LPS and OMP affected the uterine horn, ovaries, cervix, mammary gland and supramammary lymph nodes of buffalo calves inoculated either intravenously or subcutaneously, as compared to those inoculated orally.
\end{abstract}

Key words: Pasteurella multocida B:2, lipopolysaccharide, OMP, histopathology, reproductive organs, pre-pubertal buffaloes 
Pasteurella multocida is a Gram negative capsulated coccobacillus, which is primarily a secondary invader in pneumonia of cattle, swine, sheep, goats and other species (Carter \& Wise, 2003). Serotypes B and $\mathrm{E}$ are the most common and can cause haemorrhagic septicaemia, which is an acute disease principally affecting cattle and water buffalo in tropical and subtropical regions (Hirsh et al., 2005). The ability of $P$. multocida to cause diseases in different species of animals is mainly due to its specific virulence factors, e.g. LPS, capsule, adhesions and outer membrane proteins (OMP), which allow the bacteria to invade the host cells (Boyce et al., 2010).

LPS (lipopolysaccharide) is an integral outer membrane component of Gramnegative bacteria, with a critical role in the disease process by interacting directly with innate host immune defense (Reatz \& Whitefield, 2002). It confers serum resistance and is a major virulence determinant of $P$. multocida. The OMP enhances the colonisation by the bacterium by binding to host extracellular matrix proteins like fibronectin. It also serves as bacterial protective surface antigen and was reported to have a remarkable heterogenicity in different strains isolated from different animal species (Wilson et al., 2011; Wilson \& Ho, 2013). The OMP also plays an important role in the hosts' immune response to the bacterium and have been used to develop vaccines against the bacterium (Hatfaludi et al., 2010). In a recent study, P. multocida outer membrane vesicles (OMVs); OmpA, OmpH, and P6 were shown to ellicit immune response in mice. However, for conferment of complete immunity, immunisation with both P. multocida and M. hemolytica was recommended (Roier et al., 2013).
Currently, in Malaysia, P. multocida is generally controlled by the use of killed whole cell vaccines and the case fatality is high during the raining season, when most outbreaks occurred (Jesse et al., 2013). Based on our earlier studies on mice model, we observed significant pathological responses in different organs of mice inoculated with $P$. multocida surface proteins. Incidentally, we also observed significant lesions in the reproductive system (Abdullah et al., 2015). Thus, since there is paucity of literature on the pathology associated with inoculation of these surface components (LPS and OMP) in the natural host, this study was designed to fulfill that, with a particular focus on the reproductive system of the female buffalo.

Fifteen clinically healthy, non-pregnant female buffalo calves of approximately 8 months of age were selected and divided into five groups of three animals each. The buffaloes were acclimatised for 2 weeks prior to the experiment and anthelmintic (Ivomectin, Suffolk, UK) treatment was administered subcutaneously at the rate of $0.2 \mathrm{mg} / \mathrm{kg}$ body weight for three consecutive days to control internal parasitism which have been shown to influence disease development (ZamriSaad et al., 2006). Concurrently, deep nasal and vaginal swabs were collected weekly from all buffaloes and examined for the presence of $P$. multocida by bacterial isolation and polymerase chain reaction methods to ensure that the calves were free from $P$. multocida (El-Eragi et al., 2001). The calves were kept in individual pens, fed cut-grass and supplemented with palm kernel based pellets at the rate of $400 \mathrm{~g} /$ calf twice daily. Drinking water was given ad libitum.

The wild type $P$. multocida type B:2 used in this study were obtained from a 
stock culture from the Veterinary Research Institute, Ipoh, Perak. Identification of $P$. multocida type B:2 was done using the Gram staining method and biochemical characterisation of oxalase, urea broth, Sulfide Indole Motility (SIM), Triple Sugar Iron (TSI) and citrate tests. To achieve a single colony of the P.multocida type $\mathrm{B}: 2$, a pure stock culture that was stored on nutrient agar slants were sub cultured on $5 \%$ horse blood agar and incubated at $37^{\circ} \mathrm{C}$ for $18 \mathrm{~h}$. Consequently, P. multocida type $\mathrm{B}: 2$ was selected to grow on Brain Heart Infusion (BHI) broth, incubated in a shaker incubator at $37^{\circ} \mathrm{C}$ for $24 \mathrm{~h}$ before the concentration was determined using McFarland Nephelometer Barium Sulfate Standards. Preparation of $10^{12}$ colony forming unit (cfu) of $P$. multocida was performed by adding distilled water onto pure cultures of $P$. multocida before the bacteria was transferred into the sterile test tubes. These sterile test tubes were then compared with McFarland standard to determine the $10^{12}$ cfu of $P$. multocida.

The lipopolysaccharide (LPS) was extracted using Intron Biotechnology LPS extraction kit. The whole cells were centrifuged for approximately $30 \mathrm{~s}$ at 13,000 rpm at room temperature. SDS-PAGE was used to confirm the absence of protein in the extracted LPS. The extraction of OMP consisted in freezing freshly harvested cell pellets for $24 \mathrm{~h}$ prior to extraction. The cell pellets were then thawed for $15 \mathrm{~min}$ on ice and were re-suspended in $10 \mathrm{~mL}$ of native lysis buffer. The cells were then incubated on ice for $30 \mathrm{~min}$ followed by centrifugation at $14,000 \mathrm{rpm}$ for $30 \mathrm{~min}$ at $4{ }^{\circ} \mathrm{C}$. Lastly, the supernatant containing the soluble fraction of the bacterial outer membrane proteins was retained and subjected to SDS-PAGE to locate the range of protein bands present in the extract.
The research was conducted in accordance with the guidelines of the Institutional Animal Care and Use Committee (IACUC), University Putra Malaysia and was approved by IACUC No: R048/2014.

At the start of the experiment, the buffalo calves were divided into five groups of three calves each. Prior to the commencement of the experiment, all buffaloes were injected intramuscularly with dexamethasone at the dose rate of $1 \mathrm{mg} / \mathrm{kg}$ for three consecutive days, in order to mimic natural stress conditions experienced by the animals in the wild. At the end of the dexamethasone treatment, buffaloes in Group 1 (LPS I.V. group) were inoculated intravenously with $10 \mathrm{~mL}$ of LPS from $10^{12}$ of $P$. multocida type $\mathrm{B}: 2$ colony, while those in Group 2 (LPS Oral group), were inoculated orally with $10 \mathrm{~mL}$ of LPS from $10^{12}$ of colony $P$. multocida type B:2 using a stomach tube. Buffaloes in group 3 (OMP Subcutaneous group) and 4 (OMP Oral group) were subcutaneously and orally inoculated with $10 \mathrm{~mL}$ of $P$. multocida OMP at $1 \times 10^{12} \mathrm{cfu}$, respectively, while those in group 5 (negative control) were inoculated with $10 \mathrm{~mL}$ of sterile phosphate buffered saline (PBS) $\mathrm{pH} 7$ and served as the negative control. During the post infection period, all buffaloes were observed for clinical signs throughout a period of 21 days.

At the end of the study, surviving buffaloes after 21 days were sedated with xylazine $(0.3 \mathrm{mg} / \mathrm{kg})$ euthanised by exsanguination for post mortem evaluation of the reproductive organs (ovary, oviduct, uterine horn, uterine body, vagina, cervix), supramammary lymph nodes and mammary gland. Organs were collected in $10 \%$ buffered formalin, processed using routine histology preparation technique and stained with hematoxylin and eosin (H\&E). 
Lesions were scored by examining duplicate slides for each animal in a group, where 6 fields per slide were observed at $200 \times$ magnification and average were calculated for each slide. The histopathological changes observed were classified into haemorrhage and congestion; necrosis and degeneration; inflammatory cell infiltration and oedema. The changes were scored as followed: 0: normal (normal tissue); 1: mild (less than 25\% tissue affected); 2: moderate (less than 50\% tissue affected) and 3: severe (more than 65\% tissue affected) as reported previously (Jesse et al., 2015). All data (mean \pm SD) were analysed using Students' t-test (IBM SPSS Statistics 22 software) with differences considered significant at $\mathrm{P}<0.05$.

The major clinical signs and symptoms observed in buffalo calves following inoculation with $P$. multocida LPS and OMP are summarised in Table 1. Hyperthermia and mild respiratory distress were observed in all inoculation groups, except the negative control group. Only buffaloes inoculated with OMP subcutaneously died after $72 \mathrm{~h}$, while all other inoculation groups survived until the end of the experimental period (21 days). Congestion of the mucous membrane was only seen in calves inoculated i.v. with LPS, and s.c. with OMP, subcutaneously.

At postmortem, the most obvious gross change observed in the reproductive organs was congestion in both the LPS and OMP inoculation groups. Congestion was mostly mild to moderate in the ovaries, oviduct, uterine horn and uterine body. In addition, there was also an enlargement of the supra-mammary lymph nodes. The negative control animals did not show any lesions at postmortem examination.

The histopathological changes observed in all the organs following inocula- tion with LPS are summarised in Table 2. Pathological evaluation showed no significant difference $(\mathrm{P}>0.05)$ in haemorrhage and congestion, and oedema between the two groups inoculated with bacterial LPS. On the other hand, there was a significantly higher distribution $(\mathrm{P}<0.05)$ of necrosis and degeneration in the uterine horn and supra-mammary glands of calves inoculated intravenously (Fig. 1). Similarly, inflammatory cell infiltration was significantly higher $(\mathrm{P}<0.05)$ in the uterine horn and mammary gland of calves from the oral inoculation group (Fig. 2).

Following inoculation with OMP, haemorrhage and congestion were significantly more pronounced $(\mathrm{P}<0.05)$ in the ovaries, cervix, vagina, mammary and supra-mammary glands of buffaloes inoculated s.c. along with a significant increase in the severity of necrosis and degeneration in the ovary, uterine horn, uterine body, mammary and supramammary glands (Table 2). Furthermore, inflammatory cell infiltration was significantly higher $(\mathrm{P}<0.05)$ in the oviduct, uterine horns, uterine body (Fig. 3), vagina and supra-mammary glands of buffaloes inoculated s.c. with bacterial OMP.

The study describes the effects of $P$. multocida type B:2 immunogens (LPS and OMP) on the reproductive system of pre pubertal female buffaloes which have not been documented so far. During the past years, it has been reported that mice are exquisitely susceptible to $P$. multocida (Dawkins et al., 1990; Hodgson et al., 2013; Wilson \& Ho, 2013). The studies made several observations using P. multocida in different strains of mice and concluded that $P$. multocida could produce $100 \%$ mortality in mice. Besides that, oral inoculation of mice with $P$. multocida type $\mathrm{B}: 2$ resulted in the development of clinical signs as seen in the intraperitoneal 
Clinical and histopathological study on reproductive lesions caused by Pasteurella multocida type B2....

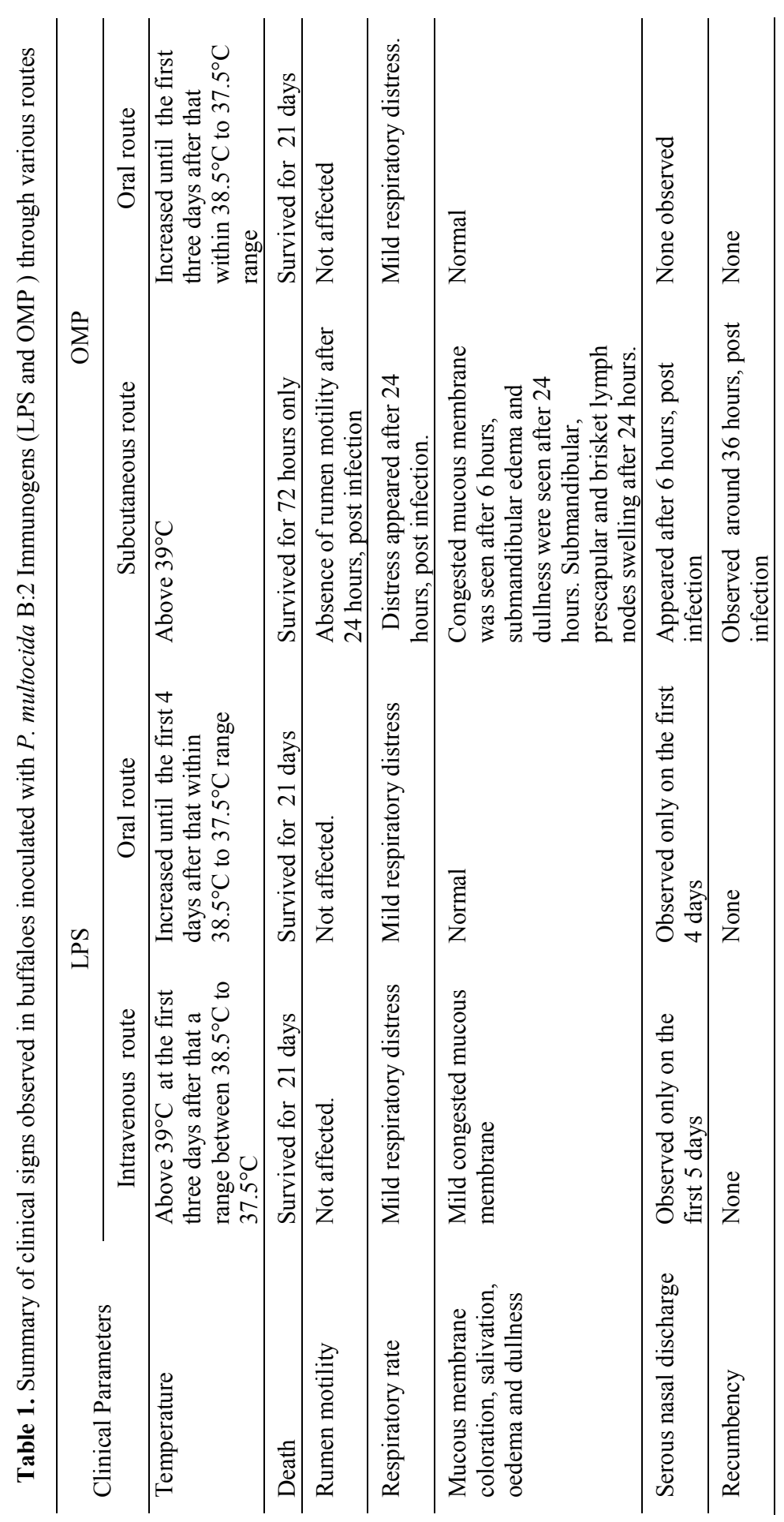


H. Ibrahim, F. Jesse, Y. Abba, E. Chung, A. Marza, A. Haron, M. Zamri-Saad, A. Omar \& A. Saharee

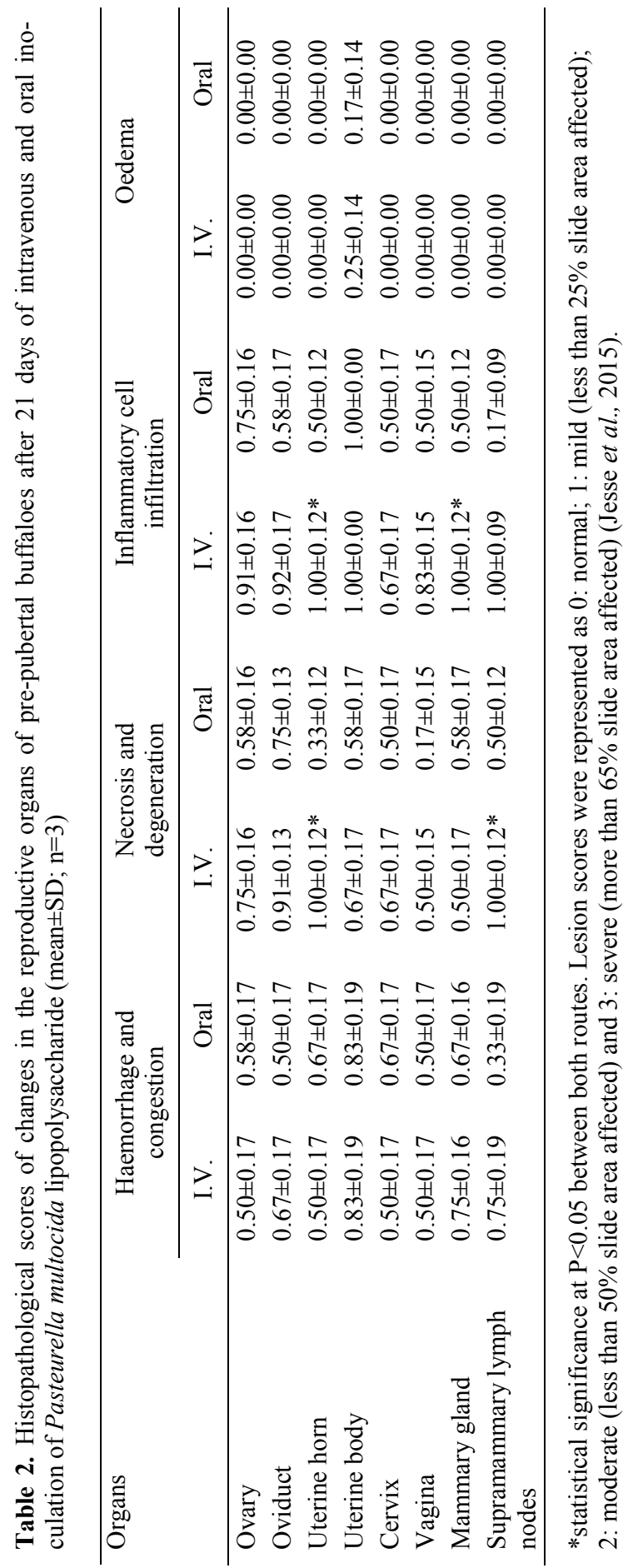

BJVM, 21, No 2 
Clinical and histopathological study on reproductive lesions caused by Pasteurella multocida type B2....

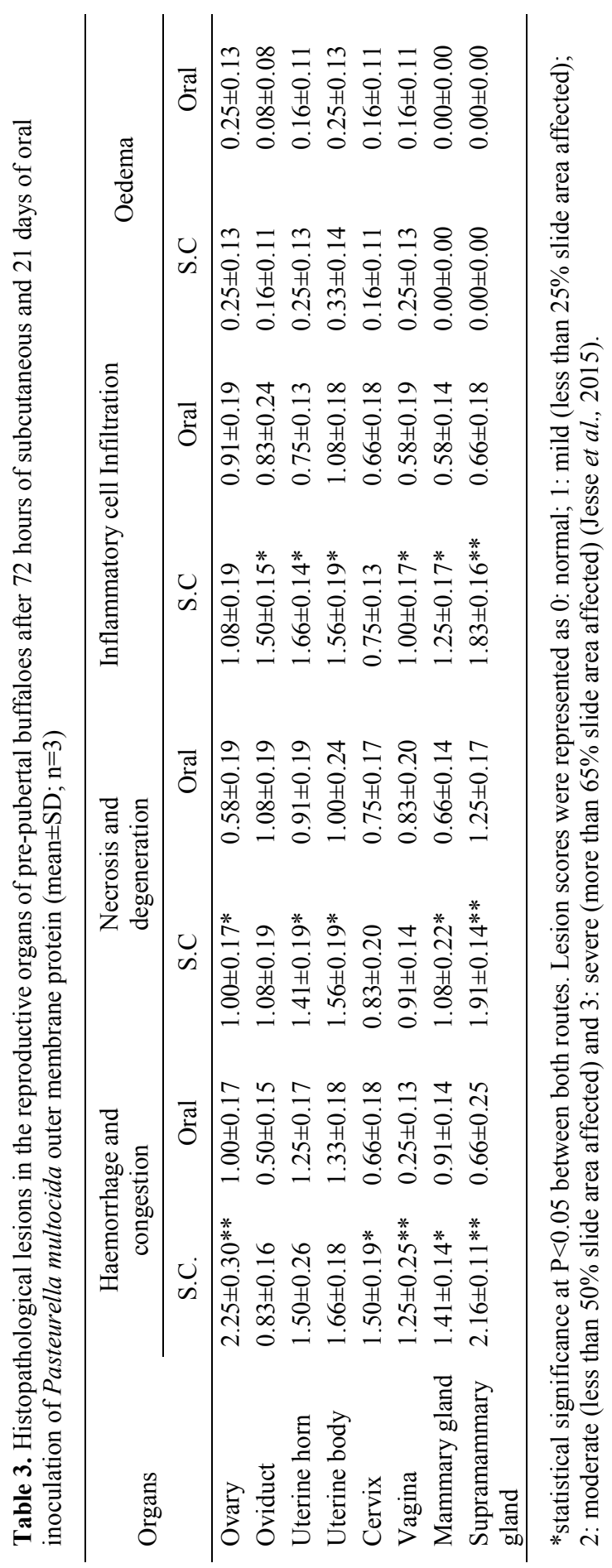


inoculation with the same organism as described in an experiment done by Dawkins et al. (1990) and Jesse (2011). Furthermore, the histopathological lesions observed were also similar to those in cattle infected naturally and experimentally with $P$. multocida type B:2 (Horadogoda et al., 1991; Khin et al., 2010).

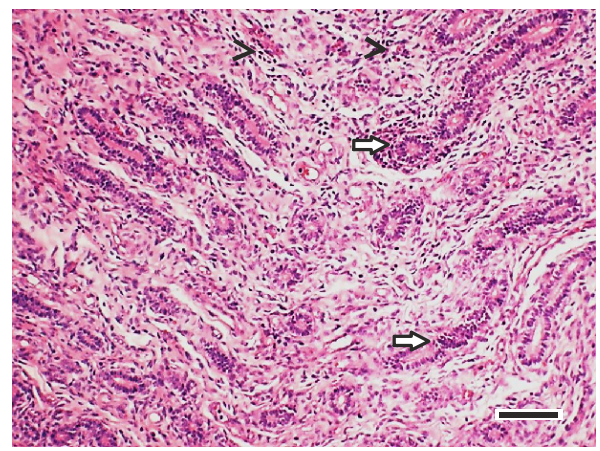

Fig. 1. Photomicrograph of the uterus (LPS intravenous inoculation), showing numerous inflammatory cells (arrowheads) and necrotic endometrial glands (arrows) in the endometrium, $\mathrm{H} \& \mathrm{E}$, bar $=100 \mu \mathrm{m}$.

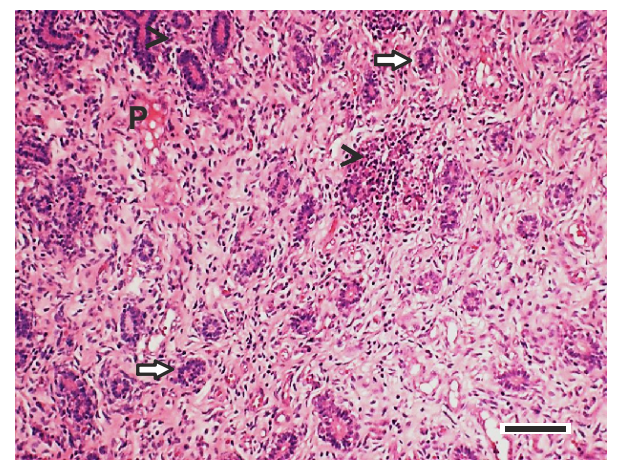

Fig. 2. Photomicrograph of the uterus (LPS oral inoculation), showing numerous inflammatory cells (arrow heads) and degenerating endometrial glands (arrows) in the endometrium, note the presence of proteinaceous matrix $(\mathrm{P})$, bar $=100 \mu \mathrm{m}$.

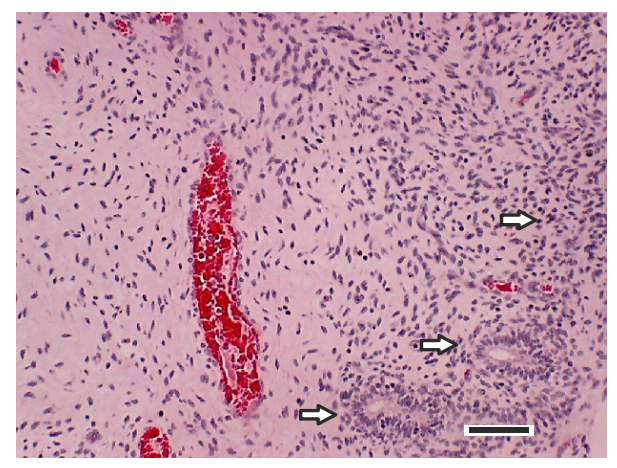

Fig. 3. Photomicrograph of the uterine body (OMP subcutaneous inoculation), showing few inflammatory cell infiltration and dilated glands in the endometrium (arrows), H\&E, bar $=100 \mu \mathrm{m}$.

In a previous study, we demonstrated the pathological lesions in the reproductive system of male and female mouse infected with the bacteria. We also observed that the testis and ovaries were among the the important organs of mice affected by $P$. multocida and its LPS (Abdullah et al., 2015). Our findings in the study were similar to a previously reported study (OIE, 2008), following oral inoculation of the bacteria in mice. In this study, we observed the uterine horn to be the most susceptible location for the LPS as evidenced by infiltration of inflammatory cells and degeneration and necrosis. We also observed that route of inoculation played a significant role in the lesion severity in the uterine horn, mammary gland and supramammary glands. Previously, Wilson \& Ho (2013) have reported the bacterial LPS to be a major virulence determinant in $P$. multocida infections; this may be the reason for the pathology observed especially following i.v. inoculation. Inoculation with the bacterial OMP showed more lethality after subcutaneous inoculation as death was recorded in all animals in the group. The literature on the 
Clinical and histopathological study on reproductive lesions caused by Pasteurella multocida type B2....

virulence of the $P$. multocida bacterial OMP is scarce; however, its ability to immunise rabbits and mice as well as its ability to evade phagocytosis by macrophages has been reported (Srivastava, 1998). This may explain the high virulence of the OMP especially following subcutaneous inoculation, since dendritic cells and macrophages are the first point of contact with the defense system. From our previous study in mice model, inoculation with $P$. multocida LPS produced more severe lesions following oral inoculation than was currently observed in the buffalo (Abdullah et al., 2015). Here, the species variation and body mass may have contributed to lesser severity observed in the buffalo. Since the i.v. inoculation has showed more severe lesions, the inoculation dose may have played a role in the oral route as well. Even though heterogeneity of Pasteurella OMP has been reported in chickens, it is yet to be extensively studied in other species, and may serve a very important and critical function in the pathogenesis of the disease in these species (Wilson \& Ho, 2013). Furthermore, extensive studies are required in order to fully understand the exact mechanisms involved in the pathogenesis of the lipopolysaccharide and outer membrane protein in the female reproductive system and its association with infertility.

This study has shown evidence of both clinical and histopathological changes in buffalo heifers inoculated with $P$. multocida LPS and OMP. The bacterial LPS has shown more severe lesions following i.v. inoculation, while the OMP was more severe following s.c. inoculation. The bacterial OMP was more lethal to buffaloes as $100 \%$ mortality was observed in the inoculated group after 72 hours.

\section{ACKNOWLEDGMENTS}

The authors would like to appreciate the staff of the Department of Veterinary Clinical Studies, Universiti Putra Malaysia. The project was funded by the Ministry of Higher Education, Malaysia (Grant No: 5524417).

\section{REFERENCES}

Abdullah, F. F. J., L. Adamu, A. Tijjani, K. Mohammed, Y. Abba, M. A. Sadiq, R. Zainal, M. J. Bin Sabu, A. A. Saharee \& A. W. Haron, 2015. Hormonal and histopathological alterations in pituitary glands and reproductive organs of male and female mice orally inoculated with Pasteurella multocida Type B:2 and its lipopolysaccharides. American Journal of Animal and Veterinary Sciences, 10, 23-35.

Boyce, J., M. Harper, I. Wilkie \& B. Adler, 2010. Pasteurella. In: Pathogenesis of Bacterial Infections in Animals, ed J. F. Prescott, Blackwell Publishing, IA, USA.

Carter, G. R. \& D. Wise, 2003. Essentials of Veterinary Bacteriology and Mycology, $6^{\text {th }}$ edn, Iowa State Press, pp. 149-152.

Dawkins, H. J. S., R. B. Johnson, T. L. Spencer \& B. Adler, 1990. Pasturella multoci$d a$ infections in mice with reference to haemorrhagic septicaemia in cattle and buffalo. Immonology and Cell Biology, 68, 57-61.

El-Eragi, A. M. S., M. M. Mukhtar \& S. H. Babiker, 2001. Specific antibodies of Pasteurella multocida in newborn calves of vaccinated dams. Tropical Animal Health Production, 33, 275-283.

Hatfaludi, T., K. Al-Hasani, J. D. Boyce \& B. Adler, 2010. Outer membrane proteins of Pasteurella multocida. Veterinary Microbiology, 144, 1-17.

Hirsh, D. C., J. Mac Lanchlan \& R. L. Walker, 2005. Veterinary Microbiology, $2^{\text {nd }} e d n$, Blackwell Publishing, pp. 87-88.

Hodgson, J. C., M. P. Dagleish, L. Gibbard, C. W. Bayne, J. Finlayson, G. M. Moon \& M. Nath, 2013. Seven strains of mice as 
potential models of bovine pasteurellosis following intranasal challenge with a bovine pneumonic strain of Pasteurella multocida A: 3; comparisons of disease and pathological outcomes. Research in Veterinary Science, 94, 634-640.

Horadagoda, N. U., M. C. L. De-Alwis, T. G. Wijewardana, K. Belak, A. U. I. Gomis \& A. A. Vilulasiri, 1991. Experimental haemorrhagic septicaemia in buffalo calves. In: Proceedings of the Fourth International Workshop on Haemorrhagic Septicaemia, Sri Lanka, pp. 11-15.

Jesse, F. F. A, 2011. Clinopathologic changes associated with Pasteurella multocida B:2 infection and its bacterial lipopolysaccharides and outer membrane protein in mice and calves. PhD Thesis, Universiti Putra Malaysia.

Jesse, F. F. A., L. Adamu, Y. O. Abdinasir, Z. Zakaria \& R. Abdullah, 2013. Clinicopathological responses of calves associated with infection of Pasteurella multocida type B and the bacterial Lipopolysaccharide and outer membrane protein immunogens. International Journal of Animal and Veterinary Advances, 5, 190-198.

Jesse, F. F. A., Y. Abba, A. Tijjani, M. A. Sadiq, M. Konto, L. Adamu, A. H. Wahid, M. L. MohdAzmi, L. T. C. Eric, M. F. Ab Rahman, N. B. Mydin \& A. A. Saharee, 2015. Gonado-hypophyseal lesions and reproductive hormonal changes in Brucella melitensis-infected mice and its lipopolysaccharides (LPSs). Comparative Clinical Pathology, DOI: 10.1007/s00580015-2133-9.

Khin, M. N., M. Zamri-Saad \& M. M. Noordin, 2010. Pathological changes in the lungs of calves following intratracheal exposure to Pasteurella multocida B:2. Pertanika Journal of Tropical Agricultural Science, 33, 113-117.

OIE Terrestial Manual, 2008. Haemorrhagic Septicaemia, Chapter 2.4.12, pp. 739-751.
Raetz, C. R. \& C. Whitfield, 2002. Lipopolysacharide endotoxins. Annual Review on Biochemistry, 71, 635-700.

Roier, S., J. C. Fenninger, D. R. Leitner, G. N. Rechberger, J. Reidl \& S. Schild, 2013. Immunogenicity of Pasteurella multocida and Mannheimia haemolytica outer membrane vesicles. International Journal of Medical Microbiology, 303, 247-256.

Srivastava, S. K, 1998. Outer membrane protein of Pasteurella multocida serotype B:2 is immunogenic and antiphagocytic. Indian Journal of Experimental Biology, 36, 530 532.

Wilson, B. A. \& M. Ho, 2013. Pasteurella multocida: From zoonosis to cellular microbiology. Clinical Microbiology Reviews, 26, 631-655.

Wilson, B. A., A. A. Salyers, D. D. Whitt \& M. E. Winkler, 2011. Bacterial pathogenesis: A molecular approach, $3^{\text {rd }}$ edn, American Society for Microbiology (ASM)

Zamri-Saad, M., A. Ernie \& M. Y. Sabri,2006. Protective effect following intranasal exposure of goats to live Pasteurella multocida B:2. Tropical Animal Health Production, 38, 541-548.

Paper received 31.07.2015; accepted for publication 12.11.2015

\section{Correspondence:}

Faez Firdaus Abdullah Jesse Department of Veterinary Clinical Studies, Faculty of Veterinary Medicine,

Universiti Putra Malaysia,

Serdang, 43400, Malaysia tel: +60175550870

e-mail: jesseariasamy@gmail.com 